\title{
The Second Law of Thermodynamics and the Big Bang.
}

\section{CPT-Symmetric Universe.}

Authors: Latham Boyle, Kieran Finn, and Neil Turok.

Phys. Rev. Lett. 121, 251301. Published 20 December 2018

\author{
Recommended with a Commentary by Robijn Bruinsma, \\ Deparment of Physics and Astronomy, University of California, \\ Los Angeles, Los Angeles, CA, 90025
}

A textbook formuation of the Second Law of Thermodynamics states that when a spontaneous process takes place in a closed system, the entropy of the system either increases (if the process is irreversible) or stays the same (if the process is reversible). Since, in classical mechanics, Newton's Laws are time-reversal symmetric (leaving aside magnetic fields), a movie of a spontaneous process taking place in a system of particles that obeys Newton's Laws can be run backwards and the resulting trajectories of the particles still will constitute a solution of Newton's Laws. The Second Law is supposed to tell us which of the two movies corresponds to what will be observed in the physical world. If one replaces time-reversal symmetry with CPT symmetry, then the same principle holds in quantum field theory. The textbook formulation of the Second Law cannot be proven and is in fact wrong. Boltzmann could prove that in a gas of particles that obeyed Newtons's Laws, the quantity $H=\int f(v) \ln f(v) d^{3} v$ either decreases in time or is a constant when the system is in thermal equilibrium (the $H$-Theorem). Here, $f(v)$ is the (in principle time-dependent) probability density in velocity space. In equilibrium, $f(v)$ becomes equal to the MaxwellBoltzman velocity distribution. If we treat $H$ as minus the entropy then this would seem to be a proof of the Second Law. However, the Boltzmann $H$-Theorem is a statement about an ensemble average of systems, not about an individual system. When, in the 1960s, Alder and Wainwright carried out computer simulations on systems of hard disks and spheres, they computed the $H$ function for an individual system by setting $f(v) d^{3} v$ equal to the number $n(v)$ of particles with a velocity $v$ in a small volume $d^{3} v$ in velocity space. Call this quantity $H_{i}(t)$. Starting from a low-entropy initial state, $H_{i}(t)$ indeed initially decreases in time but once $f(v)$ approaches the Maxwell distribution, $H_{i}(t)$ occasionally increases over brief time intervals (a plot of $H_{i}(t)$ from Alder and Wainwright is reproduced in the appendix of the book Elementary Statistical Mechanics by C. Kittel). In a state of thermal equilibrium, $H_{i}(t)$ increases in time roughly as much as it decreases in time. It is constant only after an ensemble average or, by the ergodic principle, after a time average. In fact, it was shown already in 1896 by Zermelo that if you start a system from an initial state where $H_{i}(t=0)$ is large 
compared to its equilibrium value, then $H_{i}(t)$ will return to this initial value, provided you wait long enough ("Zermelo Cycle"). The textbook formulation of the Second Law is thus, in principle, wrong. Though in practice it still may work, micro-mechanical experiments by the group of Bustamante have definitely shown the failure of the textbook formulation. Strictly speaking, it should be replaced with a formulation based on the fluctuation theorem of Evans and Searles. This theorem, which can be proven exactly for classical systems both far from equilibrium and near equilibrium, gives us a quantitative value for the probability that the entropy production rate of an individual system is negative. It can be proven from the fluctuation theorem that the ensemble-averaged rate of entropy production cannot be negative. This so-called Second Law Inequality constitutes a formally exact Second Law statement.

Thermodynamics has become popular among our colleagues in field theory and cosmology. An exact formula proposed for the entropy of a black hole by Hawking and by Bekenstein - it is proportional to the surface area of the black hole - led to the so-called "holographic principle", which has become an important principle of quantum gravity. The laws of thermodynamics have been reformulated for black holes by Brandon, Hawking and Bardeen. One important advantage in this case is that one can, at least in principle, consider observing an ensemble of black holes (there appear to be a lot of them). However, if one tries to apply the Second Law to our universe as a whole, we are dealing with just one single, individual system. The Second "Law" is reduced to the empirical observation that in our local area of the universe, entropy appears to evolve from a state with lower entropy to a state with higher entropy. We might extend it a bit. If somewhere else in the observable universe the local entropy would be decreasing with time then this should show up in the emission and absorption spectra of stars and in the time-evolution of stars. In the absence of any reports to the contrary, it is reasonable to assume that in the observable universe, the entropy is increasing in time everywhere. A natural assumption for a cosmologist would be to identify the initial $t=0$ low entropy state of the universe with the big bang. Since the observable universe evolved collectively from this low entropy state, it is reasonable that the entropy production rate is positive everywhere. But there is a hitch, which can be illustrated by returning to the molecular dynamics simulations of Alder and Wainwright. Preparing a low-entropy state in a gas of particles with, for example, $N-1$ particles in a box at rest and with the remaining single particle having a non-zero velocity, would have to be done "from the outside" and this would violate the principle of time-reversal symmetry for the particle system. Similarly, the notion that a low entropy big bang state suddenly emerges from nowhere would violate CPT invariance.

Now we come to the paper by Boyle et al. who offer a radical solution that leaves CPT intact. They argue that, prior to the big bang, the universe was in a state in which the entropy did decrease in time. The big bang takes place when the entropy stops decreasing and starts increasing. This would allow the CPT principle to remain intact. The pre- and post- big bang universes roughly are each other's time-reversed images. The scenario of Boyle et al. could be compared to the observation by Alder and Wainwright of fluctuations where over a short time interval the entropy decreased followed by a period during which the entropy increased. This is an interesting idea, which this reviewer heard proposed earlier in discussions among his astronomy colleagues. However, Boyle et al. present a concrete realization. Their particular field theory resolves as well, they claim, other puzzles such as 
the cosmic asymmetry between matter and anti-matter while it also provides an explanation for dark matter. Controversially, it does away with the inflation stage of the universe, which has a lot of experimental support. Most importantly however, the paper arrives at a testable prediction about neutrinos. The neutrino particles detected by high-energy experimentalists are left-handed. Right-handed neutrinos have not been observed. They are "sterile" in the sense that they interact only via gravity and not via the strong or weak interactions. The existence of sterile neutrinos would resolve some issues in field theory. Their mass however is so far undetermined. In the model of Boyle et al, one of the right-handed neutrinos is stable. They calculated its abundance and by matching it with the observed dark matter density, they predict that it has an enormous mass $\left(4.8 \times 10^{8} \mathrm{GeV}\right)$.

While this reviewer cannot claim to be able to follow the steps in the development of the model, it certainly made him supportive of continued neutrino experiments. The discovery of the super-massive right-handed neutrino would resolve multiple fundamental problems in cosmology and its thermodynamics. It would also provide support for an intriguing speculation by the cosmologist S. Carroll, who envisions an equilibrium ensemble of paired, time-reversed universes, each having its own big bang. The modest simulations of Alder and Wainwright could turn out to be a central paradigm of cosmology! 